\title{
Comparison of Electromyographic Amplitudes of the Adductor Magnus Muscle among Three Different Clinical Testing Positions
}

\author{
Walaa M. Elsais', Walaa S. Mohammad ${ }^{2,3}$ \\ Affiliations: 'University of Salford, Centre for Health Sciences Research, Manchester, United Kingdom, ${ }^{2}$ Majmaah \\ University, College of Applied Medical Sciences, Department of Physical Therapy, Majmaah, Saudi Arabia, ${ }^{3}$ Cairo \\ University, Faculty of Physical Therapy, Department of Biomechanics, Giza, Egypt
}

Correspondence: W.S. Mohammad, Majmaah University, College of Applied Medical Sciences, Al-Majmaah, 11952, Saudi Arabia. Email:w.mohammad@mu.edu.sa

ABSTRACT Although normalizing the EMG signals is necessary for physiological interpretation and comparison between muscles and between subjects, no EMG study has investigated the standardized position to achieve maximal contraction for the adductor magnus muscle. Accordingly, it is necessary to employ a maximum voluntary isometric contraction (MVIC) position that elicits the highest activation to increase the validity of EMG studies and provide accurate comparisons between studies. Therefore, the purpose of this study was to compare the peak electromyography (EMG) of the most commonly used positions in the literature (i.e., the fully extended hip and knee, hip-flexed $45^{\circ}$, and knee at $90^{\circ}$ ) to a novel position: prone hip extension with $90^{\circ}$ knee flexion. An ultrasound imaging system was used to guide the surface EMG electrode placement on the adductor magnus (AM) muscle, for a group of ninety-four recreational runners. AM demonstrated the greatest MVIC activity in both prone and hip-flexed $45^{\circ}$ positions with no significant differences between them $(p<0.05)$. However, significant differences were displayed between the AM activities while performing the fully extended hip position and the other two positions. Based on these results, it is recommended that the prone and hip-flexed $45^{\circ}$ positions can be used to measure the MVIC of the AM interchangeably. Furthermore, the prone position can be considered to be a position of the greatest MVIC of AM, particularly when the position of hip-flexed $45^{\circ}$ is limited as well as it can be used to quantify MVIC for both AM and hip extensor muscles simultaneously.

KEY WORDS MVIC, Adductor magnus, EMG, positions

@MJSSMontenegro

ELECTROMYOGRAPHY OF ADDUCTOR MAGNUS

http://mjssm.me/?sekcija=article\&artid=199

\section{Introduction}

The adductor magnus (AM) muscle is the largest member of the hip adductor group in terms of muscle mass. Among the hip muscles, the hip adductor group contributes $22.5 \%$ of the total muscle mass of the lower extremity (Ito, 1996). Moreover, it takes up $27 \%$ of the mass of the thigh musculature (Takizawa, Suzuki, Ito, Fujimiya, \& Uchiyama, 2014). Although the AM is considered to have the second-largest physiological cross-sectional area (PSCA) among the lower limb muscles, it has the capacity to generate extension force equal to the force produced by the gluteus maximus (Ito, Moriyama, Inokuchi, \& Goto, 2003). Similarly, the PCSAs of the adductor muscles are larger than the PCSA of the gluteus medius muscle (Williams, Wilson, Daynes, Peckham, \& Payne, 2008). Although they account for a significant proportion of the muscle mass of the lower limb of the adductor, the contribution of the adductor muscles during gait still unclear.

Electromyography (EMG) is a good means of obtaining better insight into the functional performance of the AM muscle during multiple hip movements. However, normalizing the EMG signals is necessary to overcome the natural variability and to optimize the physiological interpretation and comparison between muscles and between subjects (Lehman \& McGill, 1999). Maximal isometric voluntary contraction (MVIC) is the most broadly used approach for normalizing EMG signals, which was suggested by the SENIAM and Kinesiology's

Received: February 162020 | Accepted after revision: March 152020 | First published online: Septembar 012020

(c) 2018 by the author(s). License MSA, Podgorica, Montenegro. This article is an open access article distributed under the terms and conditions of the Creative Commons Attribution (CC BY).

Conflict of interest: None declared. 
guidelines (Hermens, Freriks, Disselhorst-Klug, \& Rau, 2000). EMG amplitudes, in this method, are expressed as a percentage of the maximum neural activation of the desired muscle (Burden, Trew, \& Baltzopoulos, 2003; De Luca, 1997). This strategy is considered a powerful approach to the physiological interpretation of signals in a healthy population. However, it is essential to note that, the MVIC normalization method is influenced by the magnitude of the MVIC test and as such shows slightly lower repeatability (Ha, Cynn, Kwon, Park, \& Kim, 2013). Accordingly, it is vital to choose an MVIC position that elicits the highest activation to increase the validity of EMG studies, and provide accurate comparisons between studies (Contreras, Vigotsky, Schoenfeld, Beardsley, \& Cronin, 2015).

Although the degree of myoelectric activity produced by AM depends on the hip joint angle, there have been only a small number of studies investigating adductor EMG patterns in musculoskeletal conditions (Benn, Pizzari, Rath, Tucker, \& Semciw, 2018; Ko, Jeon, Kim, \& Park, 2019; Lovell, Blanch, \& Barnes, 2012). Various MVIC positions have been used in the literature to assess the AM activity, including the position "Hips 0", "Hips 45", and "Hips 90" flexion (Benn et al., 2018; Lovell et al., 2012). Anecdotally, the AM is more effective hip extensor than either the hamstrings or gluteus maximus (Takizawa et al., 2014); however, no study has investigated the maximum activity of AM from prone hip extension position compared to the commonly used positions. Therefore, the purpose of this investigation was to compare the maximum activity of AM muscle from prone hip extension position with the broadly used positions in literature. It is hypothesized that hip extension position would elicit the highest AM MVIC activity.

\section{Methods}

Sample Characteristics

A total of 94 healthy recreational runners participated in this study (age: $32.02 \pm 7.15$ years, height: $176.08 \pm$ $7.05 \mathrm{~cm}$, mass $71.36 \pm 8.88 \mathrm{~kg}$, BMI: $22.95 \pm 1.81 \mathrm{~kg} / \mathrm{m} 2$ ). Runners were eligible to participate in the current study if they were between 20 to 40 years old (This age range was carefully chosen to represent the young, athletic population for whom the outcomes of the study are most likely to be implemented). In addition, participants have to be free from any neuromuscular, cardiopulmonary, musculoskeletal injury, particularly in the groin region, for a minimum of one year before joining the present study. The study was also limited to participants with a body mass index (BMI) below 25. Before testing, a decision was taken to exclude any data collected from participants with subcutaneous fat thickness more than $2 \mathrm{~cm}$ over the inner thigh as this could negatively affect the quality of EMG signals. Participants who did not meet the aforementioned criteria were excluded from the study. All participants gave their written informed consent statement in accordance with the declaration of the University of Salford Research, Innovation, and Academic Engagement Ethical Approval Panel.

\section{Measuring Devices}

There is no clear guideline for surface EMG electrode placement for AM. Moreover, the individual hip adductor muscles are close to each other at the upper medial aspect of the thigh (Watanabe, Katayama, Ishida, \& Akima, 2009). Additionally, AM is a deep muscle that occasionally appears at the superficial layers of the thigh. Therefore, electrode placement over this muscle using traditional approaches such as visual or palpation methods can be difficult. Furthermore, small errors in electrode placement could increase the possibility of cross-talk, defined as picking up signals from an adjacent muscle rather than the muscle over which the electrode is placed. Therefore, a MyLab70 (Esaote, USA) ultrasound imaging system was used to locate the $\mathrm{AM}$ and to place the surface EMG electrode accurately.

To precisely locate AM in its most superficial position and to standardize the ultrasound probe-positioning between participants, the following protocol was followed. Initially, the distance from the greater trochanter of the femur to the lateral knee joint line was measured, and $60 \%$ of this distance was used as a guide to identify the muscle position (Figure 1). Then, an ultrasound probe (LA923) of $10 \mathrm{~cm}$ length was used to map out the boundaries of the posterior border of the gracilis, AM and anterior border of the medial hamstring muscle following the procedure described by Watanabe et al. (2009). This process involves putting a water-soluble transmission gel over the participant's skin and then using a probe to image the underlying muscle structures. The position of the EMG electrode was then marked using a water-based (non-toxic) marker pen on the skin in the middle of the muscle belly (i.e., between the posterior border of gracilis and anterior border of the medial hamstring) and along the length of the muscle. Finally, a disposable adhesive Ag/ AgCl EMG electrode shaped in a figure-of-eight, and measuring $2.2 \times 4 \mathrm{~cm}$, with $1 \mathrm{~cm}$ in diameter conductive circles and $2 \mathrm{~cm}$ separating each electrode was placed over the marked area after proper skin preparation.

\section{Testing positions}

Following the electrode placement process, A DTS sensors (model 542) were attached to the surface electrode with EMG lead (542AP). This set was finally connected to a Direct Transmission System of 16 channels (Noraxon USA Inc., model 586 Tele Myo DTS Desk Receiver). EMG data were sampled at $3000 \mathrm{~Hz}$, and software (Model 131 MyoResearch-XP) was used to collect the EMG amplitudes. Two adductor testing positions described by Lovell et al. (2012) were performed in the current study. It is proposed that these two positions attain the highest EMG amplitudes for the adductor muscles. An additional testing prone hip extension position was added to test the role of AM as a strong hip extensor. Each tested position is illustrated in Table 1. 


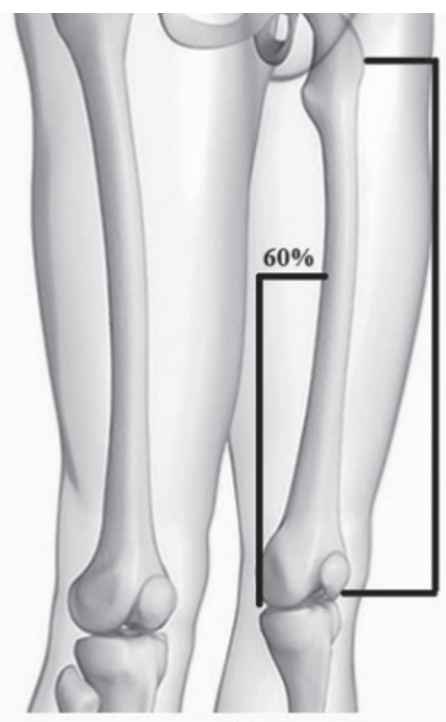

Step 1
Greater

trochanter

$100 \%$

\section{Laterarl knee}

joint line

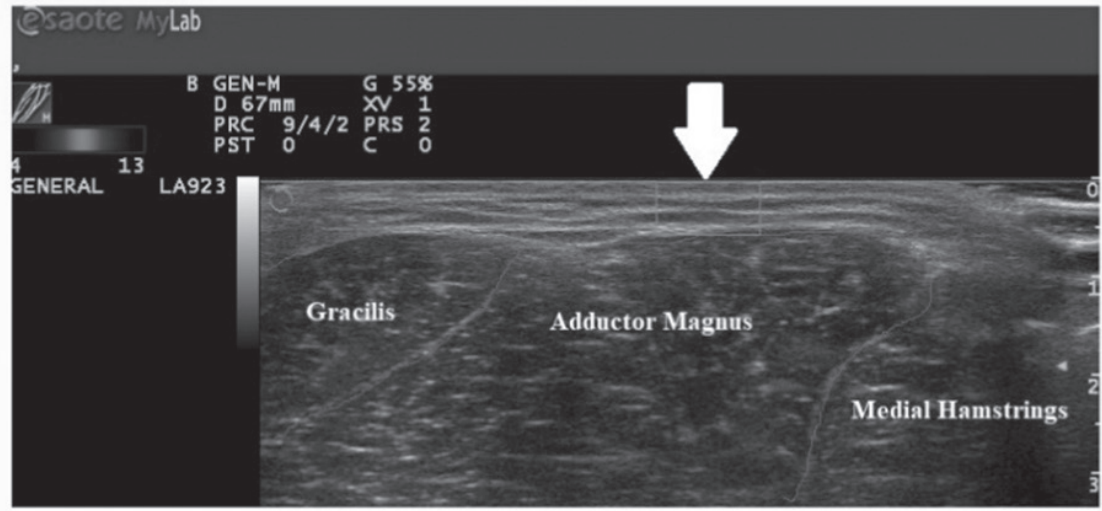

Step 2

FIGURE 1. The process of adductor magnus (AM) location. Firstly, $60 \%$ of the femur length was used as a starting point to look for the AM using a probe of ultrasound on the medial aspect of the thigh (Step 1). Then, a clear definition of all muscle boundaries around AM (Step2). This followed by placing the EMG electrode centrally on the participant's skin (white arrow).

In each testing position, the participants were asked to perform three MVIC of the tested muscle for three seconds. They also were instructed to push as hard as they could in each testing position against the manual resistance with consistent verbal encouragement throughout the whole testing process. A minimum of a minute was given as rest period between each muscle contraction to eliminate the effect of fatigue.

TABLE 1. Description of testing positions

Tested positions

Position 1

Position 2

Position 3

\section{Description and manual resistance given by the investigator}

The participant laid down on his back with fully extended hip and knee; then he was asked to adduct both his legs maximally against manual resistance.

The participant assumed laying position while his hip at $45^{\circ}$ and his knee at $90^{\circ}$. Similar to Position 1, the participant was instructed to adduct his both thighs against manual resistance maximally.

The participant was asked to lay on his stomach with $90^{\circ}$ knee flexion; then he was instructed to lift his leg toward the ceiling against manual resistance.

\section{Data processing}

The data were exported as a C3D to MATLAB for processing with custom-written software in MATLAB. There were three steps to process the raw EMG data. The first step used a high-pass filter $(20 \mathrm{~Hz})$ to remove movement artefacts and noise, as the typical frequency range of cable motion artefacts is between 1 and 50 $\mathrm{Hz}$ (Clancy, Morin, \& Merletti, 2002). The second step was rectification and envelope detection, which made all signals positive. The final step was a low-pass filter $(6 \mathrm{~Hz})$, which was used by (Hubley-Kozey, Deluzio, Landry, McNutt, \& Stanish, 2006; Hubley-Kozey, Hatfield, Wilson, \& Dunbar, 2010; Winter \& Yack, 1987) to create a linear envelope, as recommended for EMG processing for dynamic tasks (Hermie J Hermens et al., 1999). Using a filter of $6 \mathrm{~Hz}$ maintained at least $95 \%$ of signal power (Shiavi, Frigo, \& Pedotti, 1998). Following 
EMG processing, the data were exported to a Microsoft Excel 2016 spreadsheet to obtain a final result. The average RMS EMG signal was calculated separately from the middle one second of each of the three MVICs. The largest of the three values for each testing position was chosen for the tested muscle for each participant. Then, the peak value of the three tested position was used to normalize the peak EMG recordings from the other testing positions.

Statistical analysis. One-way repeated-measure ANOVAs were performed after checking normality using the Kolmogorov-Smirnov test in the statistical analysis was carried out with the Statistical Package for the Social Sciences (SPSS) (IBM SPSS Statistics 25). Post hoc comparisons were performed with the Bonferroni test. The level of statistical significance was set at $\mathrm{P}<0.05$.

\section{Results}

Table 2 displays the normalized peak EMG for the different testing positions for the AM muscle. The AM demonstrated greatest MVIC amplitude in Positions 2 and 3, which were significantly greater than in Position $1(\mathrm{p} \leq 0.001)$. In addition, there was no significant difference in AM MVIC between positions 2 and 3 (p $=1.000)$ (Figure 2).

$T A B L E 2$. The mean $\pm S D$ of normalized peakEMG amplitudes of adductor magnus during each test position

\begin{tabular}{ccccc} 
& Position 1 & Position 2 & Position 3 & P value \\
\hline Adductor magnus & $0.57 \pm 0.24$ & $0.85 \pm 0.22$ & $0.82 \pm 0.21$ & $\mathrm{p} \leq 0.001^{*}$ \\
\hline
\end{tabular}

Note. SD - standard deviation; * - Significant, $p<0.05$.

\section{Discussion}

The purpose of this study was to compare MVIC obtained from AM at different testing positions in healthy subjects. To date, there are no complete datasets that have reported the best position associated with the highest MVIC of AM muscle. Therefore, this is the first study to report on this potential link considering the different action of the AM. The primary finding of this investigation was that a position of prone with knee flexion demonstrated MVIC activation values nearly equal to that produced by the traditional position used (hip at $45^{\circ}$ ) with no significant difference between them. However, the Hip $0^{\circ}$ position presented the lowest MVIC activation values. The results from the current study are partially consistent with Lovell et al. (2012) who found that EMG activation was highest when the hip at $45^{\circ}$ position for $\mathrm{AM}$, but no direct comparison can be made because of the different positions performed (prone position not included). Moreover, it has been reported by Ko et al. (2019) that no significant difference between the activation of the AM and GMax and hamstring muscles during prone position. Possible mechanisms that explain this result are discussed below.

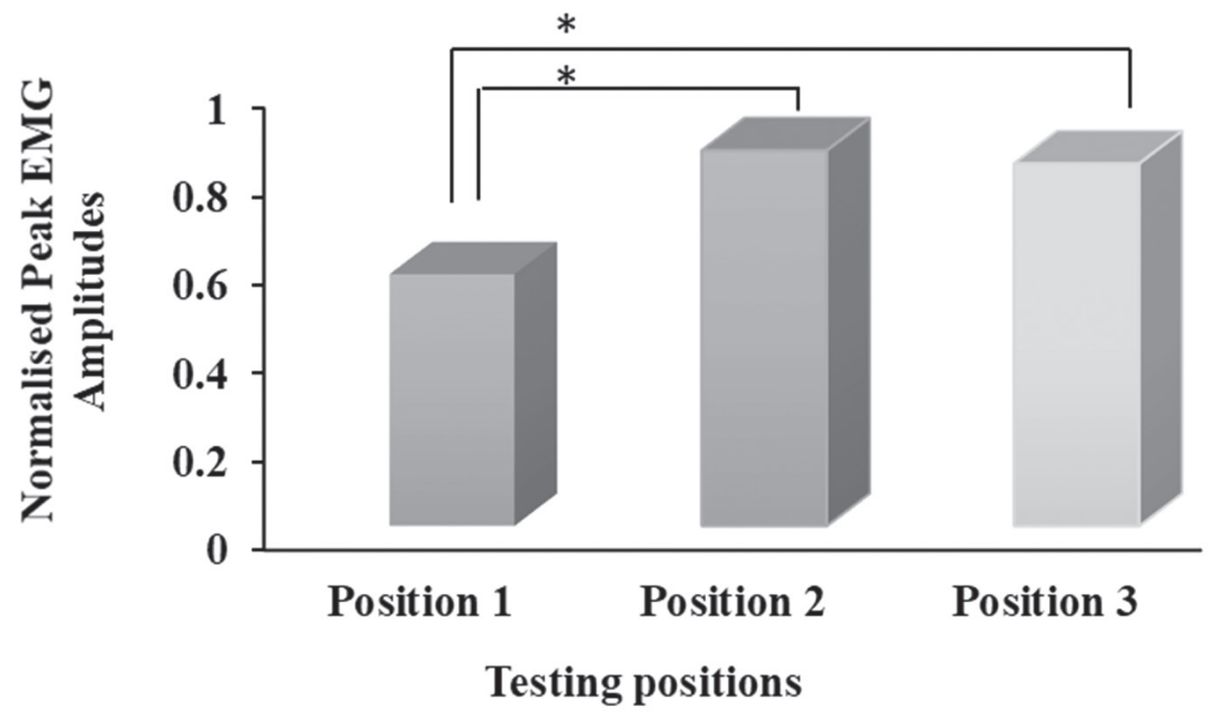

FIGURE 2. Comparison of normalized peak EMG amplitudes of adductor magnus during fully extended hip and knee (Position 1), hip-flexed $45^{\circ}$ and knee at $90^{\circ}$ (Position 2), and prone hip extension with $90^{\circ}$ knee flexion (Position 3) positions.

The AM is dissected into four parts based on courses of the corresponding perforating arteries from the deep femoral artery. Each portion of the AM may have its distinct role depending on its dynamic circumstances. The AM is divided into a hamstrings part and adductor part. The hamstrings part attaches to the adductor tubercle at the distal end of the femur (designed for stabilizing the hip joint), while the adductor part corresponds to the remaining three portions attached to the linea aspera of the femur (function as displacers for moving the thigh through an extensive range of motion) (Takizawa et al., 2014). The mass distribution and 
dissection of AM into four anatomical parts which refers as a hamstring part and adductor part suggested a various role in hip adduction during gait, and this is supported by the EMG signals in the various testing positions. It does seem plausible that the AM, due to its hip extension role, would be more active in the prone position as it may be contributing to the hip extension.

Interestingly, in the current study, no significant difference exists between the MVIC activation of the AM at prone (position 3) and when the hip at $45^{\circ}$ (position 2). This may be due to the recording site of EMG signals, which was placed over the hamstrings part of AM (located with the guidance of ultrasonography). The results of the present study could, therefore, be used to obtain the MVIC of AM from prone (position 3) when the position of hip at $45^{\circ}$ is limited, for example, during the early stage of adductor-related groin injuries. Therefore, Position 3 can be used interchangeability with Position 2 and can be used as a position to produce MVIC for both AM and Gluteus maximum (GMax) muscle.

The results of the current study showed that the MVIC values for the extended position for hip and knee joints (Position 1) significantly lower than that of the more flexed position of hip at $45^{\circ}$ (Position 2 ) and the prone position. However, the moment arm length of the AM at flexed hip position is greater than that at extended position (Németh \& Ohlsén, 1985). Recent research found that the posterior fibres of the AM muscle (where EMG electrode is placed) have the greatest moment arm for hip extension relative to other hip muscles in the extended hip position (Ko et al., 2019). Moreover, regardless of hip position, the posterior fibres of the adductor magnus are powerful extensors of the hip, similar to the hamstring muscles (Neumann, 2010). The current study is limited to a specific cohort (fit, lean runners). This choice was made in order to decrease the subcutaneous fat layer to its minimum level, thus, decreasing the effect of the fat layer on EMG signals to a minimal level. Further studies are needed to explore the position that produces the highest MVIC EMG activity of AM in females. However, a previous study suggested gender differences presented in the fat percentage of lower limb (Yamauchi, Kurihara, Yoshikawa, Taguchi, \& Hashimoto, 2015). Another limitation of this study that of measuring the activity of the superficial fibres of AM using surface EMG. Fine wire EMG is recommended to measure the EMG activity of the deep layers of AM. However, the application of fine-wire EMG is technically difficult and not widely used in clinical settings (Takizawa et al., 2014).

\section{Conclusion}

Specific to male runners, the prone and supine position with hip-flexed at $45^{\circ}$ can be used to measure the MVIC of the AM interchangeably. The results of the current study suggest that clinicians could consider the prone position as a position of greatest MVIC of AM, particularly while rehabilitating the groin-related injuries. In addition, the prone position can be used to quantify MVIC for both AM and GMax muscles in the same testing position. Future research could use different populations, such as athletic females, and also test deeper layers of AM.

\section{Acknowledgements}

The author would like to thank Deanship of Scientific Research at Majmaah University for supporting this work under Project Number No. R-1441-102.

\section{References}

Benn, M. L., Pizzari, T., Rath, L., Tucker, K., \& Semciw, A. I. (2018). Adductor magnus: An EMG investigation into proximal and distal portions and direction specific action. Clinical Anatomy, 31(4), 535-543.

Burden, A., Trew, M., \& Baltzopoulos, V. (2003). Normalisation of gait EMGs: a re-examination. Journal of Electromyography and Kinesiology, 13(6), 519-532.

Clancy, E. A., Morin, E. L., \& Merletti, R. (2002). Sampling, noise-reduction and amplitude estimation issues in surface electromyography. Journal of Electromyography and Kinesiology, 12(1), 1-16. doi:10.1016/ S1050-6411(01)00033-5

Contreras, B., Vigotsky, A. D., Schoenfeld, B. J., Beardsley, C., \& Cronin, J. (2015). A comparison of two gluteus maximus EMG maximum voluntary isometric contraction positions. PeerJ, 3, e1261.

De Luca, C. J. (1997). The use of surface electromyography in biomechanics. Journal of applied biomechanics, 13(2), 135-163.

Ha, S.-m., Cynn, H.-s., Kwon, O.-y., Park, K.-n., \& Kim, G.-m. (2013). A reliability of electromyographic normalization methods for the infraspinatus muscle in healthy subjects. Journal of human kinetics, 36(1), 69-76.

Hermens, H. J., Freriks, B., Disselhorst-Klug, C., \& Rau, G. (2000). Development of recommendations for SEMG sensors and sensor placement procedures. Journal of Electromyography and Kinesiology, 10(5), 361-374. doi:10.1016/s1050-6411(00)00027-4

Hermens, H. J., Freriks, B., Merletti, R., Stegeman, D., Blok, J., Rau, G., . . Hägg, G. (1999). European recommendations for surface electromyography. Roessingh research and development, 8(2), 13-54.

Hubley-Kozey, C. L., Deluzio, K. J., Landry, S. C., McNutt, J. S., \& Stanish, W. D. (2006). Neuromuscular alterations during walking in persons with moderate knee osteoarthritis. Journal of Electromyography and Kinesiology, 16(4), 365-378. doi:10.1016/j.jelekin.2005.07.014

Hubley-Kozey, C. L., Hatfield, G. L., Wilson, J. L. A., \& Dunbar, M. J. (2010). Alterations in neuromuscular patterns between pre and one-year post-total knee arthroplasty. Clinical Biomechanics, 25(10), 995-1002. 
doi:10.1016/j.clinbiomech.2010.07.008

Ito, J. (1996). Morphological analysis of the human lower extremity based on the relative muscle weight. Okajimas folia anatomica Japonica, 73(5), 247.

Ito, J., Moriyama, H., Inokuchi, S., \& Goto, N. (2003). Human lower limb muscles: an evaluation of weight and fiber size. Okajimas Folia Anatomica Japonica, 80(2-3), 47-55.

Ko, H.-i., Jeon, S.-y., Kim, S.-h., \& Park, K.-n. (2019). Comparison of hip extensor muscle activity including the adductor magnus during three prone hip extension exercises. Physiotherapy theory and practice, 35(5), 451-457.

Lehman, G. J., \& McGill, S. M. (1999). The importance of normalization in the interpretation of surface electromyography: A proof of principle. Journal of Manipulative and Physiological Therapeutics, 22(7), 444-446. doi:10.1016/S0161-4754(99)70032-1

Lovell, G. A., Blanch, P. D., \& Barnes, C. J. (2012). EMG of the hip adductor muscles in six clinical examination tests. Physical Therapy in Sport, 13(3), 134-140. doi:10.1016/j.ptsp.2011.08.004

Németh, G., \& Ohlsén, H. (1985). In vivo moment arm lengths for hip extensor muscles at different angles of hip flexion. Journal of Biomechanics, 18(2), 129-140.

Neumann, D. (2010). Kinesiology of the musculoskeletal system: foundations for rehabilitation. St. Louis, MO: Mosby Elsevier.

Shiavi, R., Frigo, C., \& Pedotti, A. (1998). Electromyographic signals during gait: criteria for envelope filtering and number of strides. Medical and Biological Engineering and Computing, 36(2), 171. doi:10.1007/ BF02510739

Takizawa, M., Suzuki, D., Ito, H., Fujimiya, M., \& Uchiyama, E. (2014). Why adductor magnus muscle is large: The function based on muscle morphology in cadavers. Scandinavian Journal of Medicine \& Science in Sports, 24(1), 197-203. doi:10.1111/j.1600-0838.2012.01466.x

Watanabe, K., Katayama, K., Ishida, K., \& Akima, H. (2009). Electromyographic analysis of hip adductor muscles during incremental fatiguing pedaling exercise. European Journal of Applied Physiology, 106(6), 815-825. doi:10.1007/s00421-009-1086-6

Williams, S. B., Wilson, A. M., Daynes, J., Peckham, K., \& Payne, R. C. (2008). Functional anatomy and muscle moment arms of the thoracic limb of an elite sprinting athlete: the racing greyhound (Canis familiaris). Journal of Anatomy, 213(4), 373-382. doi:10.1111/j.1469-7580.2008.00962.x

Winter, D. A., \& Yack, H. J. (1987). EMG profiles during normal human walking: stride-to-stride and intersubject variability. Electroencephalography and Clinical Neurophysiology, 67(5), 402-411.

Yamauchi, J., Kurihara, T., Yoshikawa, M., Taguchi, S., \& Hashimoto, T. (2015). Specific characterization of regional storage fat in upper and lower limbs of young healthy adults. Springerplus, 4(1), 402. 